\title{
Model for a-Si:H/c-Si interface recombination based on the amphoteric nature of silicon dangling bonds
}

\author{
Sara Olibet, ${ }^{*}$ Evelyne Vallat-Sauvain, and Christophe Ballif \\ Institute of Microtechnology (IMT), University of Neuchâtel, Breguet 2, 2000 Neuchâtel, Switzerland \\ (Received 19 January 2007; revised manuscript received 20 April 2007; published 23 July 2007)
}

\begin{abstract}
The performance of many silicon devices is limited by electronic recombination losses at the crystalline silicon (c-Si) surface. A proper surface passivation scheme is needed to allow minimizing these losses. The surface passivation properties of amorphous hydrogenated silicon (a-Si:H) on monocrystalline $\mathrm{Si}$ wafers are investigated here. We introduce a simple model for the description of the surface recombination mechanism based on recombination through amphoteric defects, i.e. dangling bonds, already established for bulk a-Si:H. In this model, the injection-dependent recombination at the a-Si:H/c-Si interface is governed by the density and the average state of charge of the amphoteric recombination centers. We show that with our surface recombination model, we can discriminate between the respective contribution of the two main mechanisms leading to improved surface passivation, which is achieved by (a) the minimization of the density of recombination centers and (b) the strong reduction of the density of one carrier type near the interface by field effect. We can thereafter reproduce the behaviors experimentally observed for the dependence of the surface recombination on the injection level on different wafers, i.e., of both $p$ and $n$ doping type as well as intrinsic. Finally, we are able to exploit the good surface passivation properties of our a-Si:H layers by fabricating flat heterojunction solar cells with open-circuit voltages exceeding $700 \mathrm{mV}$.
\end{abstract}

DOI: 10.1103/PhysRevB.76.035326

\section{INTRODUCTION}

Increasing the efficiency of crystalline silicon $(\mathrm{c}-\mathrm{Si})$ solar cells requires the reduction of both bulk and interface recombination. Even if bulk recombination is almost suppressed, the symmetry of the crystal lattice is disturbed at the surface and hence, due in particular to nonsaturated bonds (dangling bonds), a large density of defects exists. Thus, in this case, the free-carrier lifetimes are no longer limited by the quality of the bulk c-Si, but by its surface. To keep the recombination losses at the c-Si surface at minimal levels, the surface must be electronically well passivated. This is accomplished by (a) minimizing the recombination center density and/or (b) reducing strongly the density of one carrier type near the interface, as achieved by field effect. After the historical development of the metal-oxide-semicondutor field-effect transistor, which was only made possible thanks to the highquality thick passivation layer $\left(\mathrm{SiO}_{2}\right)$, metal-insulatorsemiconductor photovoltaic devices using very thin silicon dioxide $^{1}\left(\mathrm{SiO}_{2}\right)$ and silicon nitride ${ }^{2}\left(\mathrm{SiN}_{x}\right)$ passivation layers were introduced. The microscopic nature of the interface defects responsible for surface recombination has long since been identified as dangling bonds, ${ }^{3,4}$ where recombinationspecific properties are determined by the overlying passivating material. Nowadays, on state of the art, high performance devices, the standard c-Si surface passivation scheme uses thermal $\mathrm{SiO}_{2}$ yielding outstanding characteristics. ${ }^{5}$ For less demanding applications or if long-term stability is an important issue, industrial passivation schemes use $\mathrm{SiN}_{x}$. However, $\mathrm{SiN}_{x}$ layers have been observed to posses permanent positive charges, ${ }^{6}$ and this property limits their application domain. An alternative to these two major passivation processes is to use low temperature grown (typically $200{ }^{\circ} \mathrm{C}$ ) hydrogenated amorphous silicon (a-Si:H). ${ }^{7}$ In the case of photovoltaic applications, the passivation of both $\mathrm{c}-\mathrm{Si}$ wafer surfaces (i.e., the emitter and the rear surface) is of crucial importance for
PACS number(s): 73.20.At, 72.20.Jv, 73.61.Jc, 73.40.Lq

good performances. ${ }^{8}$ The current major application of the a-Si:H passivation scheme on c-Si for photovoltaic applications consists of a double-layer stack composed of an intrinsic plus a doped a-Si:H layer. Such devices are called heterojunction silicon solar cells. The application of this concept (to the emitter as well as to the rear) allowed for mass fabrication of excellent $\mathrm{c}-\mathrm{Si}$ based solar cells by Sanyo. ${ }^{9}$

So far, no satisfying simple model allows a proper description of the passivation properties on a wide range of wafer doping ( $p, n$, and intrinsic). The purpose of this paper is to introduce a simple model for the measured injectiondependent excess carrier density at the a-Si:H/c-Si interface based on a recombination mechanism via amphoteric defects, i.e., Si dangling bonds. Modeling is compared to experimental data for validation and, finally, the performances of this passivation scheme are exploited in heterojunction devices. For a complete validation of our model, thickness series of intrinsic and microdoped a-Si:H layers, as well as stacked double layers of intrinsic plus doped a-Si:H layers, are grown on c-Si wafers of both $p$ and $n$ doping type as well as on intrinsic c-Si. Overall, the various measured injection dependencies of a-Si:H/c-Si interface recombination can be fairly well reproduced by our model. More precisely, by modeling the data, we are able to quantify the two main model parameters:

(a) the dangling bond density, whose increase leads to increased recombination;

(b) the charge density at the interface, related to the magnitude of the field effect passivation.

The limits of validity of our simplified model are pinpointed on selected samples, where some discrepancies between measured data and fits are observed.

Finally, the most direct way to validate the quality of the a-Si:H passivation scheme is to complete heterojunction silicon solar cell devices: Their open-circuit voltage $\left(V_{O C}\right)$ is 
ultimately limited by the a-Si:H/c-Si interface recombination rate. By understanding and optimizing passivation properties of a-Si:H on c-Si, we are thus able to demonstrate high $V_{O C}$ 's in completed solar cells.

\section{DANGLING BOND INTERFACE RECOMBINATION MODEL}

In this work, we extend the model previously established (but not widely spread) for bulk a-Si:H recombination ${ }^{10,11}$ to the description of the surface recombination through dangling bonds. For a better understanding of our approach, the relevant underlying hypotheses and properties of this recombination model are summarized below. Although the recombination-specific parameters of the bulk dangling bonds, i.e., the capture cross sections may be different from the surface dangling bond capture cross sections, we find here that the values of the four capture cross sections considered in our model are similar for surface and for bulk dangling bonds. A plausible explanation of this observation stands from the fact that the defects at the a-Si:H/c-Si interface have the same intrinsic properties as they have in bulk a-Si:H too.

\section{A. Bulk a-Si:H recombination model}

For an analytical resolution of the injection dependence of the recombination rate, one needs a closed-form expression of the recombination rate $R\left(\mathrm{~cm}^{-3} \mathrm{~s}^{-1}\right)$. Shockley, Read, and Hall (SRH) have basically considered one discrete recombination center in the gap, having two charge conditions. Simmons and Taylor extended this SRH formalism to the case of a continuous distribution of states within the gap, ${ }^{12}$ as in disordered semiconductors. They have concluded that, as long as the capture cross sections of the continuous distribution of electronic states do not vary as a function of their energy level and as long as one can neglect the thermal reemission from them, the general form for $R$ is almost the same as for one discrete recombination center level. However, in order to discriminate between the two roles that electronic states within the gap can play, that is, they can act as trapping or as recombination centers, Simmons and Taylor introduced the notion of demarcation levels. The position of these demarcation levels is defined by the equal probability of thermal emission and free carrier capture of an electronic state and depends on the generation rate $G$ (see Fig. 1 for visualization). Electronic states lying inbetween the two demarcation levels act as recombination centers with negligible reemission probability compared to the capture probabilities. Electronic states lying inbetween demarcation level and band edge act as trap with non-negligible thermal reemission probability. The quasi-Fermi-level concept is used in the description of nonequilibrium free excess carrier distribution when the Fermi-Dirac occupation function can no longer be used to describe the probability of occupation of electronic states.

In bulk a-Si:H, monomolecular recombination via dangling bond (DB) states is the dominant mechanism for recombination at room temperature and under low to medium

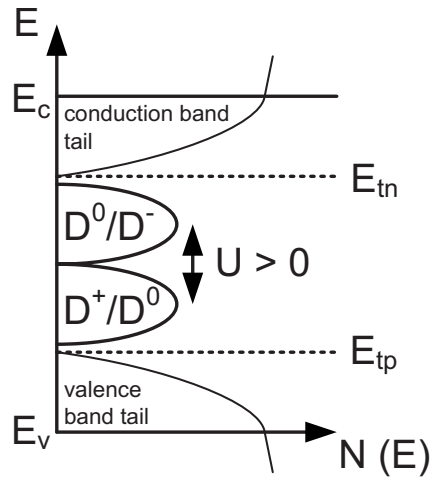

FIG. 1. One-electron representation of a continuous distribution of amphoteric recombination centers [density of states $N(E)$ ]. When unoccupied, the Si dangling bond (i.e., recombination center) $D$ will be positively charged $\left(D^{+}\right)$; when occupied by one electron, the recombination center is neutral $\left(D^{0}\right)$. These two charge conditions are represented here at the same energy level. When occupied by two electrons, the recombination center is negatively charged $\left(D^{-}\right)$, and if the correlation energy $U$ is positive, it is represented as upward shifted by $U$ (as sketched here). $E_{t n}$ and $E_{t p}$ are the demarcation levels, whose position depends on the generation rate $G$.

illumination levels. ${ }^{13}$ DB states have three different charge conditions, i.e., their occupation by zero, one, or two electrons leads to three different charge states of the $\mathrm{Si}_{3}$ sites. They can be

(1) either positively charged when not occupied by electrons $\left(D^{+}\right)$(trivalent bonded $\mathrm{Si}$ atom, $\mathrm{Si}_{3}^{+}$),

(2) or neutral when occupied by one electron $\left(D^{0}\right)$ (trivalent bonded $\mathrm{Si}$ atom with a singly occupied bond, $\mathrm{Si}_{3}^{0}$ ),

(3) or negatively charged when occupied by two electrons $\left(D^{-}\right)$(trivalent bonded $\mathrm{Si}$ atom with two electrons on the dangling bond, $\mathrm{Si}_{3}^{-}$). In this case, the energy level is shifted by an amount $U$.

The correlation energy $U$ is the energy required to place a second electron in the same $\mathrm{Si}_{3}$ orbital, and thus the energy difference between the transition levels $D^{+} / D^{0}$ and $D^{0} / D^{-}$. Hubin et al. ${ }^{11}$ found, under the conditions of a medium illumination level, a closed-form expression for $R$ where only the total density of dangling bonds $\left(N_{D B}\right)$ appears; that is, we do not need to know the shape of the continuous distribution of DB states.

Figure 1 shows the typical one-electron representation of the distribution of recombination centers in a-Si:H. In the recombination model, only transitions of free carriers to the localized dangling bond states are considered. Direct recombination between free carriers (band to band) and transitions between localized states are neglected. The demarcation levels, denoted $E_{t n}$ for electrons and $E_{t p}$ for holes, are the quasiFermi-level for traps, which means that states in the energy interval $\left[E_{t n}, E_{t p}\right]$ act as recombination centers and, therefore, not as traps. By definition, thermal emission processes from such recombination centers can be neglected if the generation rate $G$ is high enough. ${ }^{12}$ Recombination in band tails can be neglected until $G$ becomes high enough to push the demarcation levels very near the respective bands. This leads to the microscopic picture shown in Fig. 2, where two parallel recombination paths exist. Both consist of two successive capture events: 

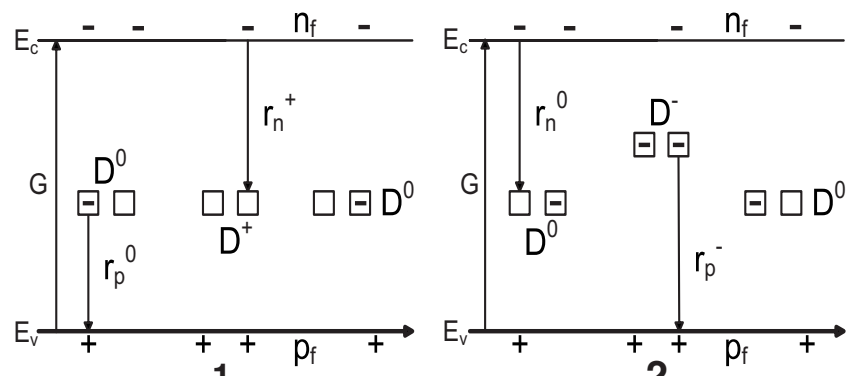

1.

2.

FIG. 2. Successive capture events leading to electron-hole recombination through $D^{0}$, i.e., when most recombination centers are in the neutral state. Two paths coexist (denoted by 1 and 2). Thermal emission processes are neglected. $n_{f}$ and $p_{f}$ are the free-carrier densities; $D^{+}, D^{0}$, and $D^{-}$are the positively, neutral, and negatively charged conditions of the dangling bonds; and $r_{p}^{0}, r_{n}^{+}, r_{n}^{0}$, and $r_{p}^{-}$are the corresponding capture rates.

(1) hole capture on a $D^{0}$ which changes the $D^{0}$ into a $D^{+}$ $\left(D^{0}+h \rightarrow D^{+}\right.$, capture rate $\left.r_{p}^{0}\right)$, followed by electron capture on this $D^{+}$, turning it again into a $D^{0}\left(D^{+}+e \rightarrow D^{0}\right.$, capture rate $r_{n}^{+}$;

(2) electron capture on a $D^{0}$ which changes the $D^{0}$ into a $D^{-}\left(D^{0}+e \rightarrow D^{-}\right.$, capture rate $\left.r_{n}^{0}\right)$, followed by hole capture on this $D^{-}$, turning it again into a $D^{0}\left(D^{-}+h \rightarrow D^{0}\right.$, capture rate $r_{p}^{-}$).

Along both paths, the recombination is limited by the less probable capture event (the smaller capture rate). Under steady-state conditions, if $r_{p}^{0}<r_{n}^{+}$, more DBs are in the neutral than in the positively charged state, and if additionally $r_{n}^{0}<r_{p}^{-}$, DBs in the negatively charged state are rare as well. If on the contrary, $r_{p}^{0} \gg r_{n}^{+}$and $r_{n}^{0} \gg r_{p}^{-}$, most DBs are either in one or the other charge state and the resulting microscopic picture is more SRH like. However, in the microscopic picture of SRH, there is only one recombination path through a recombination level having two charge conditions, i.e., neutral or negative.

Three hypotheses have to be made to find the closed-form expression for $R$ :

(1) The steady-state condition is fulfilled independently at each energy level.

(2) The illumination level is high enough so that the demarcation levels for electrons $\left(E_{t n}\right)$ and holes $\left(E_{t p}\right)$ lie outside the distribution of DB states (see Fig. 1).

(3) The capture cross sections of the DB states are independent of the energy level.

Under these three hypotheses, the calculation of $R$ can be reduced to the case of a discrete recombination level with three charge conditions. ${ }^{11}$ The resulting recombination rate can be written in terms of capture cross sections as

$$
R_{D B}=\frac{n_{f} \sigma_{n}^{0}+p_{f} \sigma_{p}^{0}}{\frac{p_{f}}{n_{f}} \frac{\sigma_{p}^{0}}{\sigma_{n}^{+}}+1+\frac{n_{f}}{p_{f}} \frac{\sigma_{n}^{0}}{\sigma_{p}^{-}}} v_{t h} N_{D B},
$$

where $N_{D B}$ is the total density of dangling bonds, $v_{t h}$ is the thermal velocity, $n_{f}$ and $p_{f}$ are the densities of free electrons and holes, respectively, $\sigma_{n}^{0}$ and $\sigma_{p}^{0}$ are the capture cross sec- tions of the neutral states, and $\sigma_{n}^{+}$and $\sigma_{p}^{-}$are the capture cross sections of the charged states. For $n_{f} \approx p_{f}$, the larger the ratio $\sigma^{+} / \sigma^{0}$, the most of DBs are in the neutral state, and the more the difference between $R_{D B}$ and $R_{S R H}$ is pronounced: When the two conditions $\sigma_{n}^{+} / \sigma_{p}^{0} \gg p_{f} / n_{f}$ and $\sigma_{p}^{-} / \sigma_{n}^{0} \gg n_{f} / p_{f}$ are fulfilled simultaneously, the expression for $R_{D B}$ reduces to

$$
R_{D B}=\left(n_{f} \sigma_{n}^{0}+p_{f} \sigma_{p}^{0}\right) v_{t h} N_{D B},
$$

which means that the recombination rate is limited by the larger free-carrier density which is opposite to the SRH case. From now on this range will be called the " $\mathrm{DB}_{i}$ domain." Conversely, for the SRH expression, the recombination is limited by the lowest free-carrier density: ${ }^{14,15}$

$$
R_{S R H}=\frac{n_{f} p_{f}}{\frac{n_{f}}{\sigma_{p}}+\frac{p_{f}}{\sigma_{n}}} v_{t h} N_{t}=\left(\frac{1}{\sigma_{p} p_{f}}+\frac{1}{\sigma_{n} n_{f}}\right)^{-1} v_{t h} N_{t} .
$$

For extrinsic, sufficiently doped a-Si:H, one type of charged dangling bonds is dominant (e.g., in $n$-type doped a-Si:H, most DBs are negatively charged), and finally, Eq. (1) reduces to a SRH-like rate, and consequently, a similar microscopic picture as for SRH recombination is reached. Taking, e.g., $n$-type material, where $n_{f} \gg p_{f}$, the expressions for $R$ are similar:

(1) By DB recombination, Eq. (1) with $n_{f} / p_{f} \gg \sigma_{p}^{-} / \sigma_{n}^{0}$ reduces to $R_{D B}=p_{f} \sigma_{p}^{-} v_{t h} N_{D B}$ i.e., recombination occurs through path 2 in Fig. 2 (as $r_{p}^{0}<r_{n}^{+}$and $r_{n}^{0}<r_{p}^{-}$; if $n_{f} \gg p_{f}$, then $r_{n}^{0}$ $\gg r_{p}^{0}$ and recombination path 1 disappears).

(2) By SRH recombination, Eq. (3) with $n_{f} / p_{f} \gg \sigma_{p} / \sigma_{n}$ reduces to $R_{S R H}=p_{f} \sigma_{p} v_{t h} N_{t}$. Therefore, this range of recombination will be called the "SRH domain."

In Fig. 3, $R_{D B}$ is plotted as a function of $n_{f}$ and $p_{f}$ [see Eq. (1)]. The ratios of the capture cross sections are set to the values given in Sec. IV B, which allow the best fit of the experimental data with our model: The ratio of neutral electron to hole capture cross section $\sigma_{n}^{0} / \sigma_{p}^{0}$ is set to $1 / 20$, and the ratio of charged to neutral capture cross section is set to $\sigma_{n}^{+} / \sigma_{n}^{0}=\sigma_{p}^{-} / \sigma_{p}^{0}=500$. The chosen value for the dangling bond density $N_{D B}=5 \times 10^{15} \mathrm{~cm}^{-3}$ is within the range of the commonly accepted values for device grade a-Si:H $\left(N_{D B}\right.$ $\left.=10^{15}-10^{16} \mathrm{~cm}^{-3}\right){ }^{16,17}$ The additionally used values are $\sigma_{p}^{0}$ $=10^{-16} \mathrm{~cm}^{2}$ and $v_{t h}=2 \times 10^{7} \mathrm{~cm} / \mathrm{s}$. They are further discussed in Sec. II C.

One can note the following general trends in Fig. 3: $R_{D B}$ reaches a maximum value for $n_{f}=p_{f}$. However, if one aims at minimizing $R$ in undoped material (i.e., in material where $n_{f} \approx p_{f}$ ), one sees from Fig. 3 that there is a local minimum at high $R_{D B}$ (dark gray surface), occurring in a range of $n_{f} / p_{f}$ determined by the value of $\sigma_{n}^{0} / \sigma_{p}^{0}$. The absolute minimum value of $R_{D B}$ can be reached when $n_{f} \neq p_{f} \gg 1$ or $\ll 1$, i.e., by field effect, where by strongly reducing the density of one carrier type we reach the same microscopic picture as for extrinsic, doped a-Si:H and, thus, as for SRH recombination (white surface). In the experiments (described in Sec. IV B) used in this work for the characterization of the passivating properties of various amorphous silicon layers, the ratio $n_{f} / p_{f}$ varies with time. At first (high-injection level), $n_{f}$ 


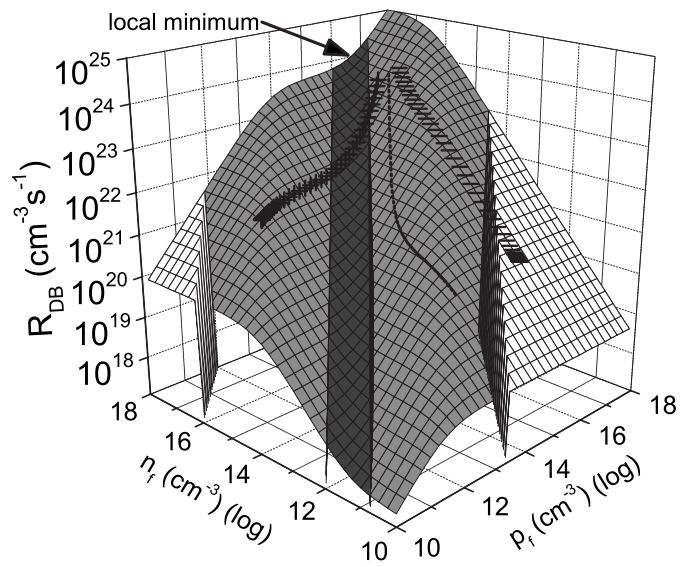

FIG. 3. Recombination rate $R_{D B}$ in a-Si:H as a function of the free-carrier densities $n_{f}$ and $p_{f}$. In this plot, capture cross-section ratios are set to $\sigma_{n}^{0} / \sigma_{p}^{0}=1 / 20$ and $\sigma_{n}^{+} / \sigma_{n}^{0}=\sigma_{p}^{-} / \sigma_{p}^{0}=500$. Over the gray surface, the recombination cannot be described by a SRH-like recombination rate; it has to be described by Eq. (1) and is therefore called the "DB domain." In its center, the dark gray surface denotes the $\mathrm{DB}_{i}$ domain, where Eq. (1) can be simplified to Eq. (2); i.e., recombination is dominated by majority carriers and, thus, opposite to the common SRH recombination. Out of the gray surface, the white surface is the SRH domain, where the recombination rate $R_{D B}$ reduces to a $R_{S R H^{-}}$like rate; i.e., the recombination process is limited by minority carriers. The trajectories on this surface plot are discussed in Sec. II C.

$\approx p_{f}$. As time proceeds, the illumination level is either decreased exponentially (quasi-steady-state method) or it is switched off (transient method). As a consequence of recombination, $n_{f} / p_{f}$ then varies with time, and $R_{D B}$ varies accordingly, remaining over the surface plot of Fig. 3, as, e.g., in the shown trajectories.

\section{B. Surface recombination calculation}

The disturbance of the symmetry of the c-Si crystal lattice at the wafer's surface results in localized dangling bonds, whose electronic state levels lie within the band gap. By growing a-Si:H on the wafer surface, the density of DBs is reduced, but is, nevertheless, supposed to be larger than in the subsequent bulk a-Si:H layer. ${ }^{18,19}$ However, a detailed knowledge of the characteristic depths over which recombination takes place within the passivating layer is lacking. Therefore, we shall reduce the abrupt a-Si:H/c-Si interface plus the subsequent passivating layer thickness where free carriers from the wafer can still recombine to what we call further on the "interface:" Consequently, we reduce the three-dimensional interface recombination to a twodimensional interface recombination. The following parameters are, thus, some average of the abrupt a-Si:H/c-Si interface plus an unknown part of the a-Si:H further grown.

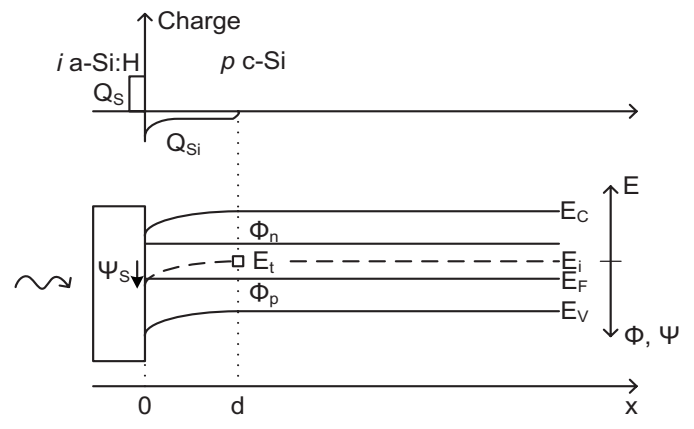

FIG. 4. Example of the charge distribution and the band diagram at the $p$ c-Si/ $i$ a-Si:H interface under illumination. Hence, we assume a unidirectional diffusion current flow from the c-Si into the intrinsic or lightly doped a-Si:H influencing the interface DB charge state. The resulting sketch is that of a small band gap insulator.

Using the same description of recombination via dangling bond states as for bulk a-Si:H, the interface recombination rate $U_{S}$ is given in analogy to Eq. (1) by

$$
U_{S}=U_{D B}=\frac{n_{s} \sigma_{n}^{0}+p_{s} \sigma_{p}^{0}}{\frac{p_{s}}{n_{s}} \frac{\sigma_{p}^{0}}{\sigma_{n}^{+}}+1+\frac{n_{s}}{p_{s}} \frac{\sigma_{n}^{0}}{\sigma_{p}^{-}}} v_{t h} N_{S}
$$

where the three-dimensional bulk DB density $N_{D B}\left(\mathrm{~cm}^{-3}\right)$ reduces to a two-dimensional interface state density $N_{S}$ $\left(\mathrm{cm}^{-2}\right)$. To describe two-dimensional recombination, commonly the interface recombination velocity $S$ is introduced, ${ }^{20}$ defined as

$$
U_{S} \equiv S \Delta n_{s},
$$

where $\Delta n_{s}$ is the excess carrier density at the interface. As a result of some field-effect passivation contribution, $\Delta n_{s}$ $=\Delta p_{s}$ is no longer valid. Thus, an effective surface recombination velocity $S_{\text {eff }}$ is defined as ${ }^{8}$

$$
S_{e f f} \equiv \frac{U_{S}}{\Delta n(x=d)},
$$

at a virtual surface within the wafer positioned at $x=d$, the edge of the c-Si surface space charge region (see Fig. 4), where the bulk c-Si photogenerated excess carrier densities obey $\Delta n(x=d)=\Delta p(x=d)$.

The interface carrier densities $n_{s}(x=0)$ and $p_{s}(x=0)$ are functions of the surface potential $\Psi_{S}$ that must be numerically determined. Assuming a unidirectional diffusion current flow from the c-Si into the a-Si:H used here (intrinsic or microdoped), the passivation layer is implicitly considered as a small band gap insulator. For this description, we use the classical drift and/or diffusion transport equation valid in $\mathrm{c}-\mathrm{Si}$, from which $n_{s}=\left(n_{0}+\Delta n\right) \exp \left(+q \Psi_{S} / k T\right)$ and $n_{s}=\left(p_{0}\right.$ $+\Delta n) \exp \left(-q \Psi_{S} / k T\right)$. The surface potential $\Psi_{S}$ is given by the nonlinear equation:

$$
Q_{\mathrm{Si}}= \pm \sqrt{\frac{2 k T n_{i} \epsilon_{0} \epsilon_{\mathrm{Si}}}{q^{2}}\left[e^{q\left(\Phi_{p}-\Psi_{S}\right) / k T}-e^{q \Phi_{p} / k T}+e^{q\left(\Psi_{S}-\Phi_{n}\right) / k T}-e^{-q \Phi_{n} / k T}+\frac{q \Psi_{S}\left(p_{0}+n_{0}\right)}{k T n_{i}}\right]}
$$


where $\Phi_{n}$ and $\Phi_{p}$ are the quasi-Fermi-levels of electrons and holes at the edge of the space charge region $(x=d)$ and are given by $\Phi_{n}=-(k T / q) \ln \left[\left(n_{0}+\Delta n\right) / n_{i}\right]$ and $\Phi_{p}=$ $+(k T / q) \ln \left[\left(p_{0}+\Delta n\right) / n_{i}\right]{ }^{8}$ These quasi-Fermi-levels depend on the nonequilibrium density of the respective carrier type (electron or hole) and are, thus, injection level dependent. In this description, $Q_{\mathrm{Si}}$ is the total image charge density induced in the c-Si. This charge density is the electrostatic image force induced by the charged defects in the a-Si:H in the interface zone as defined previously. As sketched in Fig. 4, the presence of charged defects in a-Si:H results in a freecarrier density variation in the c-Si bulk close to its surface $\left(Q_{\mathrm{Si}}\right)$. Note here that, contrary to c-Si, the major effect of ionized doping impurities in a-Si:H is to modify the average state of charge of the DBs, which results in the simultaneous variation of the free-carrier densities only for high a-Si:H doping impurity concentrations. ${ }^{21}$ In intrinsic a-Si:H, the density of free carriers and ionized impurities is negligible compared to the density of dangling bonds. Hence, our major model variable is the average state of charge of the (fixed) DBs, $Q_{S}$. Consequently, the sign of $Q_{S}$ gives the average charge state $(-$ or +$)$ of DBs within the passivating layer thickness affecting the wafer's free-carrier recombination. For simplicity, we consider only the net surface charge density $Q_{S}$, inducing the bulk image charge density $Q_{\mathrm{Si}}$ in the c-Si.

In order to keep a minimum number of parameters necessary to reproduce the experimental behavior and allow us to get a simple physical picture of recombination at the a-Si:H/ c-Si interface, the band offset between a-Si:H (band gap of about $1.75 \mathrm{eV}$ ) and c-Si (band gap of $1.1 \mathrm{eV}$ ) is not taken into account, as sketched in Fig. 4. However, for the numerical simulation of heterojunction devices, such as, e.g., SCAPS for solar cells, the discontinuities in the energy bands $E_{C}$ and $E_{V}$ have to be considered for the proper modeling of interface recombination governing charge transfer from one layer to the other in functional devices. ${ }^{22,23}$

Thermal emission processes from DB states are neglected to find the closed-form expression $R_{D B}$ in Sec. II A. However, thermal emission from recombination centers within the c-Si band gap into the a-Si:H layer cannot be neglected, as otherwise surface recombination tends to zero when $n_{s}$ and/or $p_{s}$ is very small, a situation that can occur for intrinsic or lightly doped $\mathrm{c}-\mathrm{Si}$ and which is not observed in practice (see Sec. III). Therefore, $n_{s}$ and $p_{s}$ in Eq. (4) have to be replaced by $\left(n_{s}+n_{1}\right)$ and $\left(p_{s}+p_{1}\right),{ }^{8}$ where $n_{1}$ and $p_{1}$ are the thermally emitted carrier densities from c-Si recombination centers. Recombination in the bulk of c-Si is governed by the SRH formalism; i.e., recombination occurs through centers in the gap having two charge conditions. Again, aiming to keep the model as simple as possible, we assume a single c-Si recombination center located at $x=d$ with its energy level in the middle of the gap, denoted by $E_{t}$ in Fig. 4. Therefore, we can write $n_{1}=n_{i} \exp \left[\left(E_{t}-E_{i}\right) / k T\right]$ and $p_{1}$ $=n_{i} \exp \left[\left(E_{i}-E_{t}\right) / k T\right]$, where $E_{t}$ is the energy level of this center at $x=d$. The position of the middle of the c-Si band gap at $x=d$ with respect to the middle of the a-Si:H band gap is given by $\Psi_{S}$ and, therefore, $E_{t}(x=d)-E_{i}(x=0)=-q \Psi_{S}$. The emission takes place into DBs with an assumed distri-

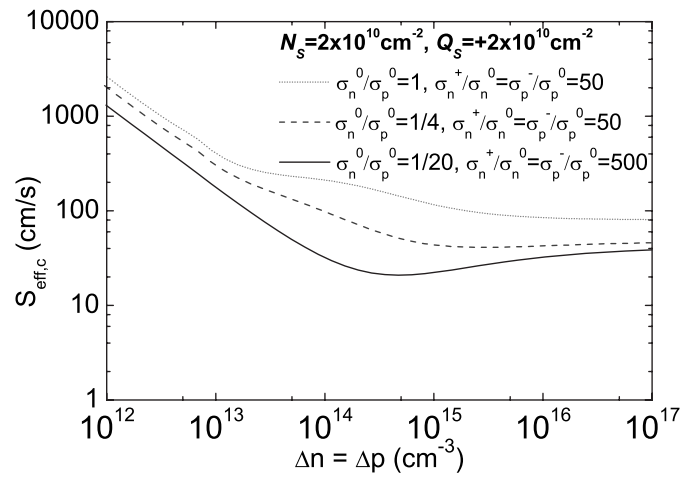

FIG. 5. Calculated behavior of $S_{e f f, c}(\Delta n)$ of a lightly $p$-type doped $(130 \Omega \mathrm{cm}) \mathrm{c}-\mathrm{Si}$ wafer passivated with a-Si:H when varying the ratios of the capture cross sections $\sigma_{n}^{0} / \sigma_{p}^{0}$ and $\sigma_{n}^{+} / \sigma_{n}^{0}=\sigma_{p}^{-} / \sigma_{p}^{0}$.

bution full width of $250 \mathrm{meV}$ (see Fig. 1). Therefore, the emission rate should stay constant above a certain absolute value for $\Psi_{S} . E_{t}-E_{i}$ is thus fixed to $-q \operatorname{sign}\left(\Psi_{S}\right)$ $\times 125 \mathrm{meV}$ for $\left|\Psi_{S}\right| \geqslant 125 \mathrm{meV}$.

Further on, the effective surface recombination velocities calculated with this model will be referred to as $S_{\text {eff,c }}$; the $c$ standing for calculation to clearly distinguish between calculated and measured values.

\section{Effect of model parameters}

First, we illustrate the effects of the microscopic recombination model parameters (i.e., the capture cross-section ratios), which will be later individually set to a unique value for all a-Si:H/c-Si combinations. By setting $\sigma_{n}^{+} / \sigma_{n}^{0}$ equal to $\sigma_{p}^{-} / \sigma_{p}^{0}$, we can reduce the model's parameter number, in agreement with most data published in the literature. ${ }^{24}$ Figure 5 illustrates the effect of varying capture cross-section ratio values on the injection level dependence of $S_{\text {eff,c }}$ on a lightly $p$-type doped $(130 \Omega \mathrm{cm}) \mathrm{c}$-Si wafer passivated with a-Si:H. In this calculation, the values of the surface recombination center density $N_{S}$ and the surface charge density $Q_{S}$ are set to $N_{S}=2 \times 10^{10} \mathrm{~cm}^{-2}$ and $Q_{S}=+2 \times 10^{10} \mathrm{~cm}^{-2}$. Note that from Eq. (4), as $\sigma_{p}^{0}$ appears in the product $N_{S} \sigma_{p}^{0}, N_{S}$ can be scaled with $\sigma_{p}^{0}$. A reasonable value of $\sigma_{p}^{0}=10^{-16} \mathrm{~cm}^{2}$ is chosen, as usual for neutral midgap states. ${ }^{25}$ The thermal velocity is set to $v_{t h}=2 \times 10^{7} \mathrm{~cm} / \mathrm{s}$, i.e., to the thermal velocity of $\mathrm{c}-\mathrm{Si}$, where $v_{t h_{n}} \approx v_{t h_{p}}$ as the effective masses of electrons and holes are only slightly different. The neutral electron and hole capture cross sections $\sigma_{n}^{0}$ and $\sigma_{p}^{0}$ are generally assumed to be in the same range. ${ }^{24}$ The dotted curve in Fig. 5 shows the calculated injection dependence of $S_{\text {eff,c }}$, with $\sigma_{n}^{0}=\sigma_{p}^{0}$ together with a value for the ratio of charged to neutral capture cross section equal to $\sigma_{n}^{+} / \sigma_{n}^{0}=\sigma_{p}^{-} / \sigma_{p}^{0}=50$ (as found by Beck et al. ${ }^{26}$ ). The dashed curve shows the calculated behavior of $S_{e f f, c}$ with the neutral electron to hole capture cross section $\sigma_{n}^{0} / \sigma_{p}^{0}=1 / 4$. This value is obtained by using the experimentally observed ratio of the a-Si:H $\mu \tau^{0}$ product $\mu_{n} \tau_{n}^{0} / \mu_{p} \tau_{p}^{0}=10,{ }^{27}$ considering a value for the ratio of mobilities in a-Si:H $\mu_{n} / \mu_{p}=3$, (Ref. 28) and the slight asymmetry in thermal velocity ratio $v_{t h_{n}} / v_{t h_{p}}=1.22$. For the solid curve, we assumed the values found to give the best fit to our 


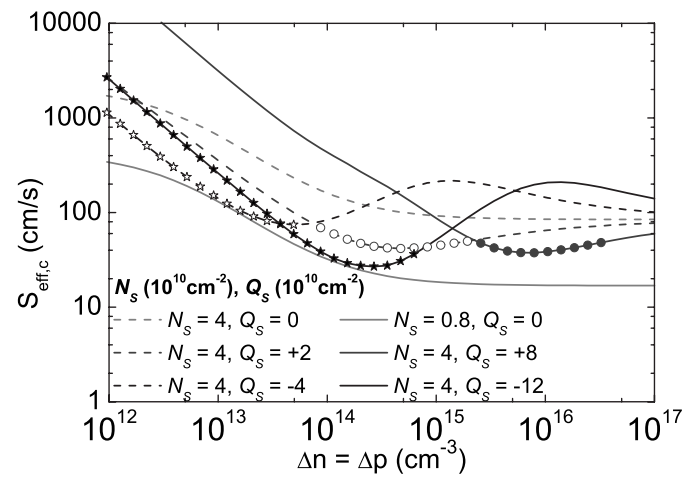

FIG. 6. Calculated behavior of $S_{\text {eff,c }}(\Delta n)$ of a lightly $p$-type doped $(130 \Omega \mathrm{cm}) \mathrm{c}-\mathrm{Si}$ wafer passivated with a-Si:H. When reducing the surface recombination center density $N_{S}, S_{\text {eff,c }}$ is reduced over the whole injection level range. Positive or negative surface charge densities induce field-effect passivation, and hence, modify the excess carrier density at which the minimal value of $S_{e f f, c}$ is reached, as well as the absolute minimum value of $S_{\text {eff,c }}$.

whole set of experimental data (see Sec. IV B), $\sigma_{n}^{0} / \sigma_{p}^{0}$ $=1 / 20$ and $\sigma_{n}^{+} / \sigma_{n}^{0}=\sigma_{p}^{-} / \sigma_{p}^{0}=500$.

The variation of the capture cross-section ratio $\sigma_{n}^{0} / \sigma_{p}^{0}$ modifies the symmetry of the surface plot in Fig. 3 and sets, thus, the local minimum of $S_{\text {eff,c }}$ with respect to $n_{f}=p_{f}$ in the curve in Fig. 5. The increase of $\sigma_{n}^{+} / \sigma_{n}^{0}=\sigma_{p}^{-} / \sigma_{p}^{0}$ from 50 to 500 widens the DB domain and, thus, enlarges the range of the local minimum of $S_{\text {eff,c }}$.

In order to illustrate the effects of the two remaining recombination model parameters $N_{S}$ and $Q_{S}$, we calculate $S_{\text {eff,c }}$ as a function of the excess carrier density with the microscopic parameters set to the values above $\left(\sigma_{n}^{0} / \sigma_{p}^{0}=1 / 20\right.$, $\left.\sigma_{n}^{+} / \sigma_{n}^{0}=\sigma_{p}^{-} / \sigma_{p}^{0}=500\right) . N_{S}$ represents the interface density of dangling bonds (recombination centers) and, thus, the quality of the passivation, whereas $Q_{S}$ is the average state of charge of the defects and is, thus, representative of the magnitude of the field effect on the passivation. Figure 6 shows again the case of a lightly $p$-type doped c-Si wafer passivated with a-Si:H. As can be seen, a reduction of the interface recombination center density $N_{S}$ shifts $S_{\text {eff,c }}(\Delta n)$ down [as $S_{\text {eff }, c}$ given by Eqs. (4) and (6) is simply proportional to $N_{S}$ ], while adding field-effect passivation by positive or negative surface charge density $Q_{S}$ increases the injection dependence of $S_{\text {eff,c }}$. These are the rough tendencies observed generally for surface passivation. ${ }^{8}$

For the purpose of comparison, the regions of the curves highlighted with stars correspond to the SRH domain in Fig. 3 . The rest of the curves belong to the $\mathrm{DB}$ domain, wherein the parts highlighted by circles belong to the $\mathrm{DB}_{i}$ domain (see Fig. 6). On a lightly $p$-type doped wafer surface, without any surface charge $Q_{S}$, one has $p_{s} \geqslant n_{s}$, and, as a result, the recombination rate never lies in the $\mathrm{DB}_{i}$ domain when $\sigma_{n}^{0} / \sigma_{p}^{0}=1 / 20$. Conversely, depending on the amount of positive surface charge density, an inversion of the $p \mathrm{c}$-Si surface $n_{s} \geqslant p_{s}$ results in a shift of $R_{D B}$ into the $\mathrm{DB}_{i}$ domain. Finally, negative surface charge density leads to hole accumulation at the $p$ c-Si surface and, thus, $p_{s} \gg n_{s}$, resulting in the recombination rate $R_{D B}$ lying in the SRH domain. The variations of $R_{D B}$ as a function of $n_{s}$ and $p_{s}$ are visualized by means of the surface plot of $R_{D B}$ in Fig. 3 for the three cases where $Q_{S}$ $=0, Q_{S}=+8 \times 10^{10} \mathrm{~cm}^{-2}$, and $Q_{S}=-8 \times 10^{10} \mathrm{~cm}^{-2}$. We observe that, whereas $n_{s}=p_{s}$ at high injection levels, at lower injection levels $n_{s}\left(p_{s}\right)$ remains constant, and this corresponds to minority-carrier-limited recombination and to a slice plane in the surface plot of $R_{D B}\left(n_{s}, p_{s}\right)$.

We conclude that the physical effect of positive (negative or neutral) dangling bond charge in the interface region allows the tuning of the magnitude of field-effect-like passivation. High surface charge densities $Q_{S}$, as resulting from a-Si:H doping or external field application, are needed to reach the microscopic picture when the dangling bond recombination rate $R_{D B}$ simplifies to the SRH-like rate. As long as lightly doped $\mathrm{c}$-Si wafers are passivated with intrinsic a-Si:H layers, the amphoteric nature of the DB states dominates recombination and, consequently, the SRH recombination model does not allow the reproduction of the experimental data.

\section{EXPERIMENT}

\section{A. Passivation layers}

a-Si:H passivation layers are grown by very high frequency plasma enhanced chemical vapor deposition at $70 \mathrm{MHz}$ and at a substrate temperature of $180{ }^{\circ} \mathrm{C}$ under standard conditions used for a-Si:H solar cell fabrication in a single chamber plasma reactor. Intrinsic (i) layers are grown with a mixture of silane $\left(\mathrm{SiH}_{4}\right)$ and hydrogen $\left(\mathrm{H}_{2}\right)$ as source gases (silane concentration $\mathrm{SC}=\left[\mathrm{SiH}_{4}\right] /\left[\mathrm{SiH}_{4}+\mathrm{H}_{2}\right]=30 \%$ ). By adding only a few ppm of doping gas [phosphine $\left(\mathrm{PH}_{3}\right)$ for $n$-type doping and trimethylborane for $p$-type doping, both diluted in $\mathrm{H}_{2}$ ] to the gas mixture used for $i$-layer growth, the average state of charge of the DBs and, thus, $Q_{S}$ can be varied without much influencing neither the dangling bond density $N_{D B}{ }^{29}$ Such low-level doping is called microdoping ( $\mu$ dop). Heavier doping results in an increase in the dangling bond density $N_{D B}$ and does, thus, no longer permit to vary $Q_{S}$ independently of $N_{D B}$. Doped a-Si:H layers are used in this work only stacked with $i$ a-Si:H layers. These configurations permit to modify the average state of charge of the DBs by the electric field imposed in the $i$ a-Si:H layers when fixing the outer surface potential. The abrupt a-Si:H/ c-Si interface of our samples and devices is verified by transmission electron microscopy. Our interfaces look similar to those published by other authors. ${ }^{30}$

The experimental technique used here for the evaluation of the surface recombination velocity requires double-sided $\mathrm{c}-\mathrm{Si}$ wafer passivation. For modeling purposes, the surface passivation has to be symmetric as well [Eq. (8) is, otherwise, not applicable]. Consequently, all the wafers studied here are sequentially passivated on both sides. Prior to a-Si:H growth, the native oxide on top of the out of box, both side polished c-Si wafers, is removed by diluted HF. After double-sided a-Si:H passivation, samples are thermally annealed at the deposition temperature $\left(180^{\circ} \mathrm{C}\right)$.

\section{B. Lifetime measurements}

In order to evaluate the interface recombination rate by $S_{\text {eff }}$, we use high-quality lightly doped, double-side polished 
float-zone wafers. The bulk carrier lifetime $\tau_{b}$ of these c-Si wafers is set to $10 \mathrm{~ms}$ (a rather overestimated value).

The overall recombination is evaluated via an effective lifetime measurement from which $S_{\text {eff }}$ can be deduced. The excess carrier density (ECD) dependent effective carrier lifetime $\tau_{e f f}$ in double-side passivated $\mathrm{c}-\mathrm{Si}$ is evaluated with a Sinton Consulting WCT-100 system with both the transient and quasi-steady-state (QSS) photoconductance techniques; ${ }^{31}$ the latter analyzed in the so-called generalized mode. In this setup, the inductively measured excess photoconductance is given by $\sigma_{L}=q\left(\Delta n_{a v} \mu_{n}+\Delta p_{a v} \mu_{p}\right) W$, where $\Delta n_{a v}=\Delta p_{a v}$ is the average ECD, $W$ the wafer thickness, and $\mu_{n}$ and $\mu_{p}$ the well known electron and hole mobilities in c-Si. $\tau_{e f f}$ is related to $\Delta n_{a v}$ by the generation rate $G_{L}$. The experimentally measured value of ECD corresponds to the model variable $\Delta n(x=d)=\Delta p(x=d)$ in Eq. (6). The transient photoconductance measurement mode consists of measuring wafer conductivity vs time after a very short and intense light pulse. This technique is only appropriate for the evaluation of photogenerated carrier lifetimes appreciably greater than the flash duration. On the contrary, in the QSS mode, the wafer conductivity vs illumination level is measured during a long, exponentially decaying light pulse, where the generalized analysis of the data allows the characterization of arbitrary lifetimes. In order to evaluate the effective interface recombination rate over the widest possible range of ECD, several measurements are combined. This is the origin of some small discontinuities present in the following measurement data. At low excess carrier densities, the data are often acquired in the transient mode. Transient and QSS data, when analyzed in the generalized mode, converge to almost identical values at higher ECDs, but in some cases bigger discrepancies occur at lower ECDs. This will be further discussed in Sec. IV D 1.

Due to the rather high lifetimes measured and the symmetrical surface passivation scheme used here, the effective surface recombination velocity $S_{\text {eff }}$ at the a-Si:H/c-Si interface is related to $\tau_{\text {eff }}$ by the simplified equation:

$$
S_{\text {eff }}=\left(\frac{1}{\tau_{\text {eff }}}-\frac{1}{\tau_{b}}\right) \frac{W}{2}
$$

All $S_{\text {eff }}$ calculated by Eq. (8) from measured $\tau_{\text {eff }}$ will be called measured $S_{e f f}$ and be referred to as $S_{e f f, m}$ further on.

Additionally, from lifetime measurements, an expected open-circuit voltage can be deduced. This value is calculated from the separation of the quasi-Fermi-levels. ${ }^{20}$

\section{RESULTS: MODELING EXPERIMENTAL DATA}

\section{A. Motivation}

In order to be able to extract criteria for the quality of interface passivation (the value of $N_{S}$ ), we want to fit the measured $S_{\text {eff, }}(\mathrm{ECD})$ with our model described by Eqs. (4), (6), and (7). For this purpose, the values of the capture crosssection ratios $\sigma_{n}^{0} / \sigma_{p}^{0}, \sigma_{n}^{+} / \sigma_{n}^{0}$, and $\sigma_{p}^{-} / \sigma_{p}^{0}$ have to be known. If one refers to values published in the literature, ${ }^{24}$ their range is so wide that this does not allow a fit of our experimental data with our model, as their influence on injection level dependence is so important. Thus, here we measure the injection level dependence of the effective surface recombination velocity and choose values which allow for a reasonable fit of all our experimental data simultaneously. Then, while keeping these values set, we analyze the $S_{\text {eff, } m}(\mathrm{ECD})$ curves as a function of $N_{S}$ and $Q_{S}$. Thus, by fitting measured $S_{\text {eff, } m}(\mathrm{ECD})$ curves with our model, we want to find the microscopic mechanism limiting the interface recombination. This knowledge will allow us to exploit the performances of this specific passivation scheme in many different applications, such as thin-film transistors and solar cells (front, back, and diffused emitters). Here, we demonstrate the performance of the a-Si:H passivation scheme by fabricating heterojunction solar cells on c-Si for both $p$ and $n$ doping types.

\section{B. Methodology}

In the whole mathematical description of our model, there is only one single equation that cannot be easily calculated. The nonlinear Eq. (7) is, therefore, solved by MATLAB. Further on, in order to evaluate the couple of model parameters $N_{S}$ and $Q_{S}$ characterizing passivation, resulting from either the reduction of interface recombination center density or from the field effect, we have to determine the capture crosssection ratios involved in Eq. (4). The best fits with our model to the experimental data, obtained for a thickness series of $i$ a-Si:H layers on lightly $p$ - and $n$-type doped as well as on intrinsic wafers, yield a capture cross-section ratio of neutral electron to hole $\sigma_{n}^{0} / \sigma_{p}^{0}=1 / 20$ and charged to neutral capture cross-section ratios $\sigma_{n}^{+} / \sigma_{n}^{0}=\sigma_{p}^{-} / \sigma_{p}^{0}=500$. These best fits were obtained by several trial and error, "manually" introducing various sets of published values for $\sigma_{n}^{0} / \sigma_{p}^{0}$ and $\sigma_{n}^{+} / \sigma_{n}^{0}=\sigma_{p}^{-} / \sigma_{p}^{0}{ }^{24}$ Once the microscopic parameters are fixed to their preevaluated values, then $N_{S}$ and $Q_{S}$, giving the best accordance between the model and the experimental data for each case, are again obtained by "manual" adjustment. Such a procedure is not time consuming here, as (see Sec. II C) $Q_{S}$ gives the shape of the $S_{\text {eff,c }}(\mathrm{ECD})$ curve while the magnitude of $N_{S}$ only shifts up or down the whole curve by a constant factor. To summarize, MATLAB calculation input parameters are the capture cross sections $\sigma_{n}^{0}, \sigma_{p}^{0}, \sigma_{n}^{+}, \sigma_{p}^{-}$and the thermal velocity $v_{t h}$, further, we search the set of c-Si/a-Si:H interface parameters $N_{S}$ and $Q_{S}$ giving a reasonable agreement between theory and experience.

First, we investigate the passivation performance of intrinsic a-Si:H on c-Si with an a-Si:H layer thickness of $5 \mathrm{~nm}$ as used in heterojunction solar cell devices. ${ }^{32}$ As such thin $i$ a-Si:H layers are never used without capping layers, we exploit the passivation performance of $i$ a-Si:H by growing $40 \mathrm{~nm}$ thick layers instead, which are slightly thicker than the typical total thickness of stacks grown for heterojunction solar cell fabrication. Further on, we change the average DB charge state of $40 \mathrm{~nm}$ thick $i$ a-Si:H layers by microdoping, thus, varying $Q_{S}$ without increasing $N_{S}$. The same additional field-effect passivation is realized by growing stacks of $i$ a-Si:H plus doped layers. Table I summarizes the couples $N_{S} / Q_{S}$ found to give best accordances between measured and calculated injection level dependent surface recombinations 
TABLE I. Model parameter couples $N_{S} / Q_{S}$ giving the best accordance between measured and calculated injection level dependent surface recombinations for various configurations of passivating layers and c-Si wafers. $\sigma_{n}^{0} / \sigma_{p}^{0}=1 / 20$ and $\sigma_{n}^{+} / \sigma_{n}^{0}=\sigma_{p}^{-} / \sigma_{p}^{0}=500$ are the same for all layers.

\begin{tabular}{|c|c|c|c|c|}
\hline $\begin{array}{l}\text { Sample } \\
\text { No. }\end{array}$ & a-Si:H layers ${ }^{\mathrm{a}}$ & Wafer & $\begin{array}{c}N_{S} \\
\left(10^{10} \mathrm{~cm}^{-2}\right)\end{array}$ & $\begin{array}{c}Q_{S} \\
\left(10^{10} \mathrm{~cm}^{-2}\right)\end{array}$ \\
\hline 1 & $5 \mathrm{~nm}$ intrinsic $(i)$ & $>15 \mathrm{k} \Omega \mathrm{cm}(n),{ }^{\mathrm{b}}$ & 12 & 6 \\
\hline 2 & $40 \mathrm{~nm} i$ & $>15 \mathrm{k} \Omega \mathrm{cm}(n)$ & 11 & 7 \\
\hline 3 & $5 \mathrm{~nm} i$ & $n 60 \Omega \mathrm{cm}, \mathrm{a}^{\mathrm{c}}$ & 7 & -9 \\
\hline 4 & $5 \mathrm{~nm} i$ & $n 60 \Omega \mathrm{cm}, 10 \mathrm{hDD}^{\mathrm{b}}$ & 17 & 1 \\
\hline 5 & $40 \mathrm{~nm} i$ & $n 60 \Omega \mathrm{cm}$ & 2 & 2 \\
\hline 6 & $500 \mathrm{~nm} i$ & $n 60 \Omega \mathrm{cm}$ & 6 & 2 \\
\hline 7 & $5 \mathrm{~nm} i$ & p $130 \Omega \mathrm{cm},{ }^{\mathrm{b}}$ & 11 & 1 \\
\hline 8 & $40 \mathrm{~nm} i$ & p $130 \Omega \mathrm{cm}$ & 4 & 2 \\
\hline 9 & $40 \mathrm{~nm} \mu \operatorname{dop} n$ & $>15 \mathrm{k} \Omega \mathrm{cm}(n)$ & 1 & -10 \\
\hline 10 & $40 \mathrm{~nm} \mu \mathrm{dop} p$ & $>15 \mathrm{k} \Omega \mathrm{cm}(n)$ & 7 & 8 \\
\hline 11 & $40 \mathrm{~nm} \mu \operatorname{dop} n$ & $n 60 \Omega \mathrm{cm}$ & 1 & -12 \\
\hline 12 & $10 \mathrm{~nm} i+40 \mathrm{~nm} n$ & $n 60 \Omega \mathrm{cm}$ & 1 & -15 \\
\hline 13 & $40 \mathrm{~nm} \mu \mathrm{dop} p$ & $n 60 \Omega \mathrm{cm}$ & 3 & 2 \\
\hline 14 & $10 \mathrm{~nm} i+30 \mathrm{~nm} p$ & $n 60 \Omega \mathrm{cm}$ & 2 & 1 \\
\hline 15 & $40 \mathrm{~nm} \mu \mathrm{dop} n$ & p $130 \Omega \mathrm{cm}$ & 4 & -4 \\
\hline 16 & $10 \mathrm{~nm} i+40 \mathrm{~nm} n$ & p $130 \Omega \mathrm{cm}$ & 1 & -9 \\
\hline 17 & $40 \mathrm{~nm} \mu \mathrm{dop} p$ & p $130 \Omega \mathrm{cm}$ & 6 & 2 \\
\hline 18 & $10 \mathrm{~nm} i+30 \mathrm{~nm} p$ & p $130 \Omega \mathrm{cm}$ & 10 & 1 \\
\hline
\end{tabular}

${ }^{a}$ Grown symmetrically onto both sides of the wafers.

${ }^{\mathrm{b}}$ After $10 \mathrm{~h}$ dark storage in ambient atmosphere.

cAnnealed.

for the various configurations of passivating layers/c-Si wafers studied experimentally here. In the resulting figures, the measured $S_{e f f, m}(\mathrm{ECD})$ is indicated by symbols, while $S_{e f f, c}(\mathrm{ECD})$ calculated with the model parameters given in Table I is indicated by lines. $\tau_{\text {eff }}(\mathrm{ECD})$ as originally acquired by the Sinton lifetime tester is indicated by the right hand side of the $y$ axis of the figures. To the axis with gray labels scaling $\tau_{\text {eff }}(\mathrm{ECD})$ for the intrinsic and lightly $n$-type doped wafers, a second scale with labels in italic is added for the lightly $p$-type doped wafer that is almost twice as thick [see Eq. (8)]. Note that as for $\tau_{b}$ a finite value of $10 \mathrm{~ms}$ is set, the simple inverse proportionality of $\tau_{\text {eff }}$ to $S_{\text {eff }}$ assumed for the right hand $y$ axis becomes invalid as $\tau_{\text {eff }}$ approaches $\tau_{b}$ and, consequently, these axes are limited to $5 \mathrm{~ms}$. Concretely, the maximum measured value for $\tau_{e f f}$ of $2.5 \mathrm{~ms}$ is, in the graphical representation by the right hand $y$ axis, overestimated by $30 \%$.

Finally, the passivation performance of asymmetrically grown stacks, i.e., $i$ a-Si:H/p a-Si:H on one side and $i$ a-Si:H/n a-Si:H on the other side, are measured as well. The deposition of metallic contacts on the top layers of these stacks results in heterojunction photovoltaic devices. The measurements of the high performances of these devices prove the validity of this passivation scheme for c-Si.

\section{Passivation by intrinsic a-Si:H layers}

Figure 7 shows the measured ECD dependent interface recombination, measured on samples consisting of $5 \mathrm{~nm}$ thick $i$ a-Si:H layers grown symmetrically on three different c-Si wafers: intrinsic, lightly doped $n$ and $p$ type. The measurements are made directly after thermal annealing as well as after $10 \mathrm{~h}$ of dark storage in ambient air (partially shown in Fig. 7). This comparison illustrates the effect of the change at the outer surface of the $i$ a-Si:H layer (e.g., postoxidation or water adsorption), leading to an ill-defined outer

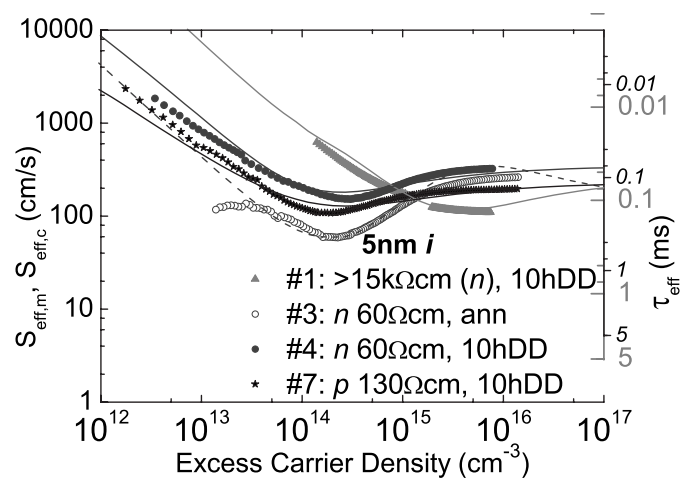

FIG. 7. Variation of the effective surface recombination velocity as a function of excess carrier density measured for intrinsic $5 \mathrm{~nm}$ thick a-Si:H layers passivating three different c-Si wafers. On the lightly $n$-type doped wafer, the $S_{\text {eff,m }}(\mathrm{ECD})$ measurements are shown directly after thermal anneal (ann) as well as after $10 \mathrm{~h}$ of dark storage in ambient air (10hDD). The symbols show the experimental values, and the lines are the best fits with parameters given in Table I. 


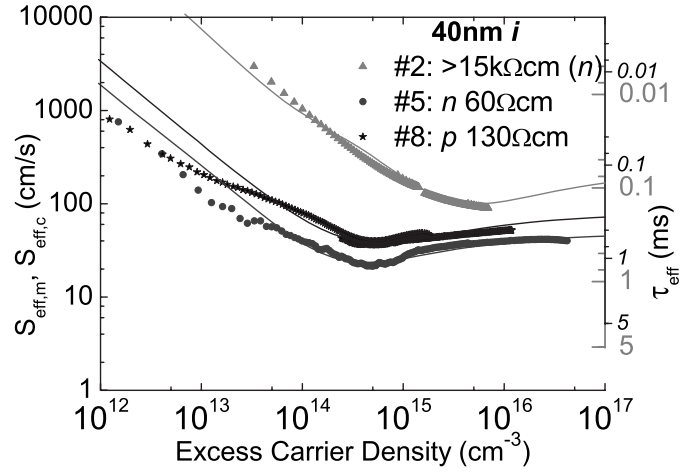

FIG. 8. Measured (symbols) and fitted (lines) variations of $S_{\text {eff }}$ as a function of ECD for intrinsic $40 \mathrm{~nm}$ thick a-Si:H layers passivating three different c-Si wafers. Fitted curves are obtained with the parameters listed in Table I.

surface potential, as verified by the corresponding value for the surface charge density $Q_{S}$ extracted from our model (see Table I, samples 3 and 4).

Because of this instability with respect to storage in air, such ultrathin $i$ a-Si:H layers, unprotected by further grown capping layers (such as a doped a-Si:H layer in heterojunction solar cells or a $\mathrm{SiN}_{x}$ layer in a c-Si solar cell ${ }^{33}$ ), are of no practical use. Thus, typical a-Si:H passivation layers used in this work are $40 \mathrm{~nm}$ thick. Their interface passivation performance on the different wafers is shown in Fig. 8. Such thicker layers permit to suppress the postoxidation effects on the a-Si:H/c-Si interface passivation. In this case, the slightly positive value obtained for $Q_{S}$ (samples 2, 5, and 8 in Table I) in the interface layers could originate from the here neglected band offset between crystalline and amorphous silicon. ${ }^{34}$ Indeed, in general, the conduction band offset is found to be smaller than the valence band offset in the literature, and this can lead to positive charged DBs at the interface.

Interestingly, the value of $N_{S}$ evaluated from our fitting procedure depends on the a-Si:H layer's thickness (see Table I: 5, 40, and $500 \mathrm{~nm} i$ a-Si:H, samples (4-6). $N_{S}$ is reduced by increasing the thickness of the passivation layer from 5 to $40 \mathrm{~nm}$. This observation is explained with a technologi-

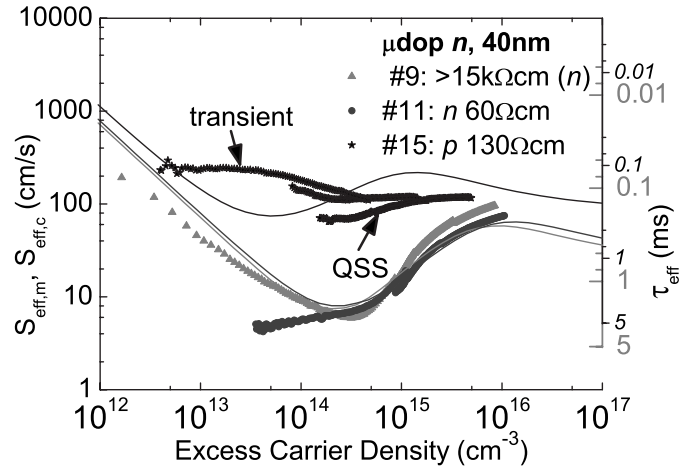

FIG. 9. $n$-type microdoped $40 \mathrm{~nm}$ thick a-Si:H layers passivating three different c-Si wafers. The symbols show the measurements, and the lines the fits with parameters from Table I of the ECD dependent $S_{\text {eff }}$.

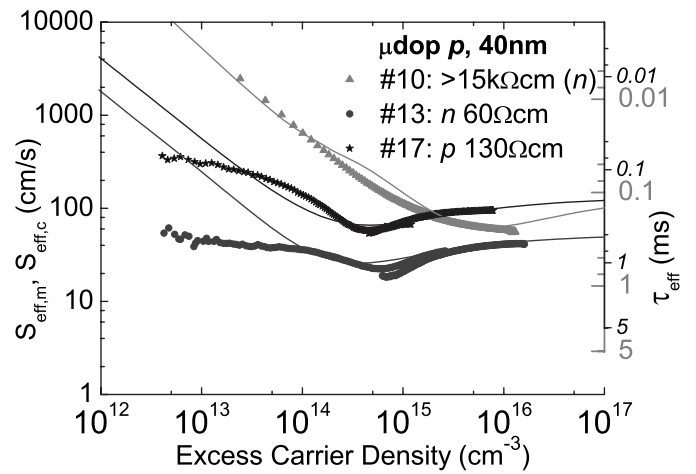

FIG. 10. Effective surface recombination as a function of ECD measured for $p$-type microdoped $40 \mathrm{~nm}$ thick a-Si:H layers passivating three different $\mathrm{c}-\mathrm{Si}$ wafers. The symbols show the measurements, and the lines the fits with parameters given in Table I.

cal phenomenon: the production of surface defects by the initial interface between the plasma and the c-Si surface. Subsequent growth of the a-Si:H layer leads, due to structural relaxation, to a reduction of this plasma-induced defects at the c-Si surface. This relaxation effect is still occurring for a-Si:H layers that are about $30 \mathrm{~nm}$ thick. ${ }^{35}$ For $i$ a-Si:H layer thickness increasing from 40 to $500 \mathrm{~nm}$, we observe again an increase of $N_{S}$ with no variation in $Q_{S}$ as seen from Table I. This more defective interface may be induced by increasing mechanical stress at the c-Si/a-Si:H interface when growing such thick layers. ${ }^{36}$

In summary, our recombination model fits well to the experimental data and allows a quite simple interpretation of the observed effects of postoxidation and thickness dependence of passivating properties of $i \mathrm{a}-\mathrm{Si}: \mathrm{H}$ layers on three different c-Si wafers.

\section{Passivation by microdoped a-Si:H layers}

Figures 9 and 10 show the dependence of $S_{\text {eff }}$ on ECD for the three different c-Si wafers passivated with microdoped $n$-type and $p$-type a-Si:H layers having a thickness of $40 \mathrm{~nm}$. A sketch of the recombination model pertaining to this case at the a-Si:H/c-Si interface is given in Fig. 11 for intrinsic c-Si.

The $n$-type microdoping leads to a strongly increased average negative state of charge of the dangling bonds within
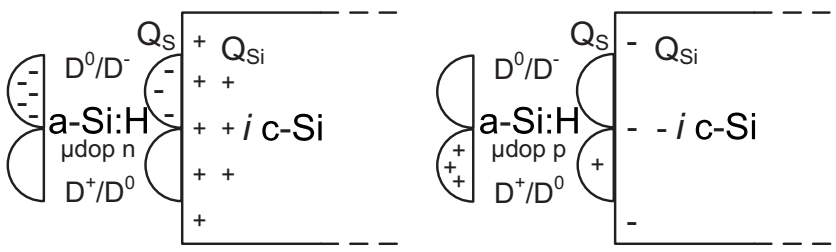

FIG. 11. Sketch of the average state of charge of the recombination centers in microdoped a-Si:H layers passivating intrinsic c-Si: $n$-type $\mu$ dop is shown on the left, $p$-type $\mu$ dop on the right (charge density is adapted to measurements). The sketched occupation level in the a-Si:H distant from the interface in the drawings illustrates the expected "bulk" occupation in microdoped a-Si:H layers. 
the passivation interface (as shows up in the values of $Q_{S}$ for samples 9, 11, and 15 in Table I). Note that, as expected, $N_{S}$ is not modified by microdoping. The experimental data measured for $n$-type microdoped a-Si:H passivation layers on lightly $p$-type microdoped c-Si show a discrepancy between the transient (low to middle illumination level) and the QSS (middle to high illumination level) measurements and are consequently poorly fitted too. How this could be related to differences in the two measurement modes is discussed in Sec. IV D 1.

For $p$-type microdoped passivating a-Si:H layers, fit parameters (i.e., $N_{S}$ and $Q_{S}$ ) do not differ much from the intrinsic ones (as can be verified with a comparison of samples 10, 13, and 17 with samples 2, 5, and 8 in Table I). The reason for this can be that the chosen microdoping level is not sufficient enough to increase further the already existing positive charge state of the DBs observed for intrinsic layers. Nevertheless, in this case, $N_{S}$ increases slightly due to the detrimental effect of boron incorporation, resulting in a more defective a-Si:H structure already observable at low-level boron doping. ${ }^{29}$

The measured injection dependence of microdoped a-Si:H/c-Si interface recombination can be reproduced by our model, but discrepancies between measured $S_{e f f, m}$ and $S_{e f f, c}$ in the low ECD range show that the limits of validity of our simplified model are reached on some samples (see, e.g., sample 11 in Fig. 9). These limits of validity are further discussed in Sec. IV D 2.

\section{Discrepancies between $S_{\text {eff,m }}$ measured in the transient and QSS modes}

The $S_{\text {eff,m }}(\mathrm{ECD})$ curve of the lightly $p$-type doped c-Si passivated by $n$-type microdoped a-Si:H layers shows large discrepancies in the curve segments acquired in the transient and QSS modes (see sample 15 in Fig. 9). We think that this might be related to the fact that during QSS measurements, although we use a filter to cut off wavelengths shorter than $700 \mathrm{~nm}$, residual photogeneration can occur in the a-Si:H, while during transient measurements there is only a carrier flow from the c-Si; i.e., there are far fewer photogenerated carriers inside the a-Si:H during transient measurements. Consequently and roughly speaking, the interface layer analyzed during the lifetime measurement would be thicker in QSS mode than in the transient mode. This also agrees with the picture of a more defective a-Si:H material at the interface: as the transient measurement analyzes some of only the more defective initial a-Si:H layer, it thus yields higher recombination $\left(S_{\text {eff,c }}\right)$ than the QSS measurement that analyzes the less defective a-Si:H too. Further work is needed, however, to understand fully the reason why the transient and QSS measurements yield the same results in the low to middle ECD range for some combinations of passivation layer and wafer type, while others do not.

\section{Low-injection-level discrepancies between the calculated and the measured $S_{\text {eff }}$}

In Figs. 9 and 10, discrepancies between the measured $S_{e f f, m}$ and the fitted $S_{e f f, c}$ can be observed in the low ECD

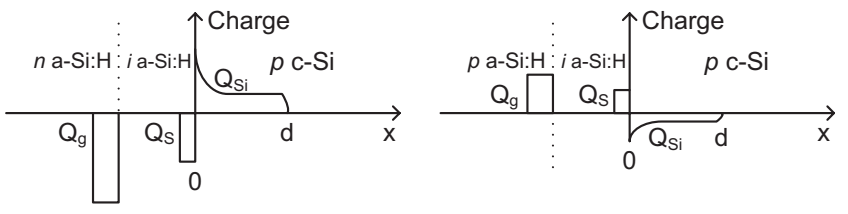

FIG. 12. Sketch of the charge distribution when passivating lightly $p$-type doped c-Si by stacks of $i / n$ a-Si:H (left hand) or $i / p$ a-Si:H (right hand).

range (most pronounced for sample 11 in Fig. 9). In fact, the increase of $S_{e f f, c}$ at low injection levels is due to the back diffusion of thermally emitted carriers out of recombination centers within the c-Si into the a-Si:H $\left(n_{1}\right.$ and $\left.p_{1}\right)$. As expected, this effect has to be taken into account when studying interface passivation of undoped or lightly doped c-Si by intrinsic a-Si:H. However, the discrepancies observed when passivating $\mathrm{c}-\mathrm{Si}$ by microdoped layers, at decreasing illumination levels, are not related with this phenomenon. It is rather the condition relating to hypothesis 2 in Sec. II A that is no longer fulfilled. The reason for this is that at low injection levels the energy interval limited by the quasi-Fermilevels for traps $\left[E_{t n}, E_{t p}\right]$, within which electronic states act as recombination centers and not as traps, does not include anymore the whole DB distribution (see Fig. 1). Thus, at low $\mathrm{ECD}$, the number of DBs acting as recombination centers is decreasing with decreasing ECD. As $S_{\text {eff,c }}$ given by Eqs. (4) and (6) is simply proportional to $N_{S}$, the not further increased $S_{\text {eff, } m}$ with decreasing injection level can, thus, be explained. In reality, the competing effects of free-carrier injection from the c-Si wafer (that leads to an increase of $S_{\text {eff,c }}$ ) and of the decreasing $N_{S}$ (that leads to a decreasing $S_{\text {eff,c }}$ ) can result in an ECD independent measured $S_{e f f, m}$ in some specific cases. In a-Si:H, the more the Fermi level is distant from midgap, the greater the chance for $E_{t n}$ or $E_{t p}$ to lie still in the DB distribution at low ECD. The occurrence or not of lowinjection-level discrepancies between the calculated and measured $S_{\text {eff }}$ is in agreement with the calculated positions of $E_{t n}$ and $E_{t p}$ on the whole set of our experimental data.

\section{E. Passivation by double-layer stacks of intrinsic and doped a-Si:H layers}

In the aim of approaching lifetime test structures to the configuration eventually used in heterojunction solar cells, we grow symmetrical stacks of $i$ a-Si:H/doped a-Si:H layers. $10 \mathrm{~nm}$ thick $i$ a-Si:H layers with interface relaxing capping layers reach low $N_{S}$. The doped layers are grown up to a thickness of several tens of nanometers and physically fix the $i$ a-Si:H potential. Figure 12 shows this by means of a sketch of the resulting charge distribution. The resulting Fermi level shift in the $i$ a-Si:H layer changes the average charge state of dangling bonds in the same manner as in the case of microdoping. This similarity is the experimental evidence that microdoping and externally imposed Fermi level shift have the same effect on a-Si:H passivating layers. The ECD dependent passivation performance of stacks of intrinsic plus doped a-Si:H layers is shown in Fig. 13 on lightly $n$-type doped c-Si and in Fig. 14 on lightly $p$-type doped c-Si. For 


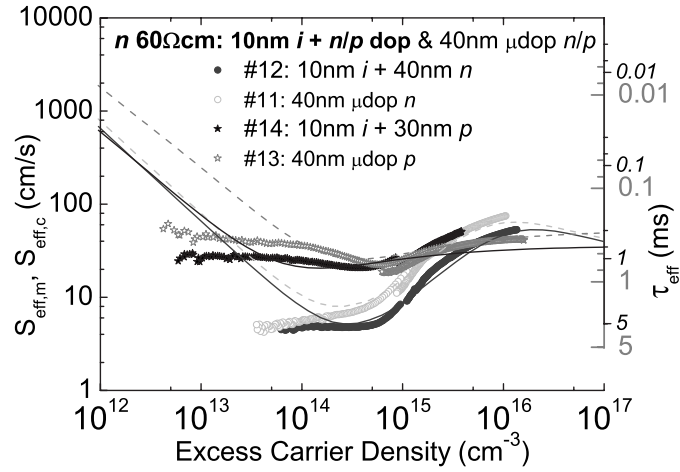

FIG. 13. Measured and calculated $S_{\text {eff }}(\mathrm{ECD})$ for the lightly $n$-type doped c-Si surface passivated by stacks of intrinsic plus doped layers. Empty symbols and dotted lines replicate the microdoped layer's passivation for comparison purpose. The symbols show the measurements, and the lines the fits with the parameters given in Table I. Microdoping the $40 \mathrm{~nm}$ thick $i$ a-Si:H layer or shifting the Fermi level in a $10 \mathrm{~nm}$ thick $i$ a-Si:H layer externally with a doped layer results in similar experimental behaviors of $S_{\text {eff }}(\mathrm{ECD})$.

purpose of comparison, the results from passivation by microdoped layers are replicated in these figures (empty symbols and dashed lines).

The corresponding fit values (samples 12 and 16 in Table I) show the expected behavior for stacks of $i / n$ a-Si:H layers; that is, $Q_{S}$ is strongly increased and negative and $N_{S}$ is unchanged.

In contrary to $p$-type microdoping, doped $p$-type layers are verified to be really doped. Furthermore, they are the same as used in our high- $V_{O C}$ heterojunction devices (see Sec. IV F). Nevertheless, $Q_{S}$ and $N_{S}$ stay as in the case of $p$-type microdoping at about the same values as for the intrinsic passivation layers (compare in Table I samples 14 and 5 and samples 18 and 8). This poor passivation performance of $i / p$ stacks is also observed by other authors. ${ }^{37,38}$

The measured injection level dependence of interface recombination of double-layer stacks of intrinsic and doped a-Si:H layers passivating lightly $p$ - and $n$-type doped c-Si is

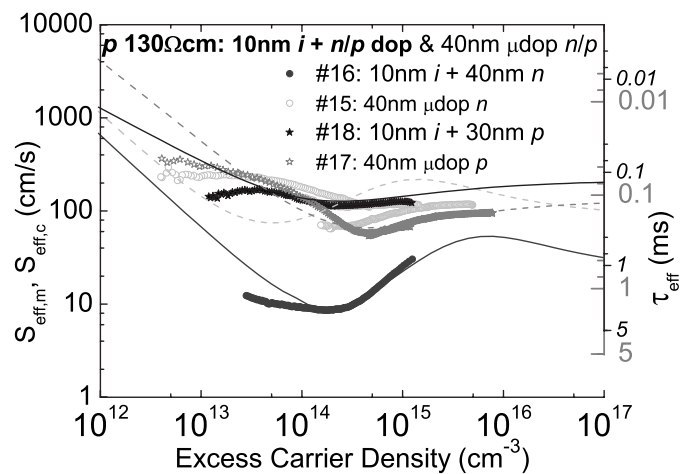

FIG. 14. Measured and calculated $S_{\text {eff }}(\mathrm{ECD})$ for the lightly $p$-type doped c-Si surface passivated by stacks of intrinsic plus doped layers. Empty symbols and dotted lines replicate the microdoped layer's passivation for comparison purpose. The symbols show the measurements, and the lines the fits with the parameters given in Table I.

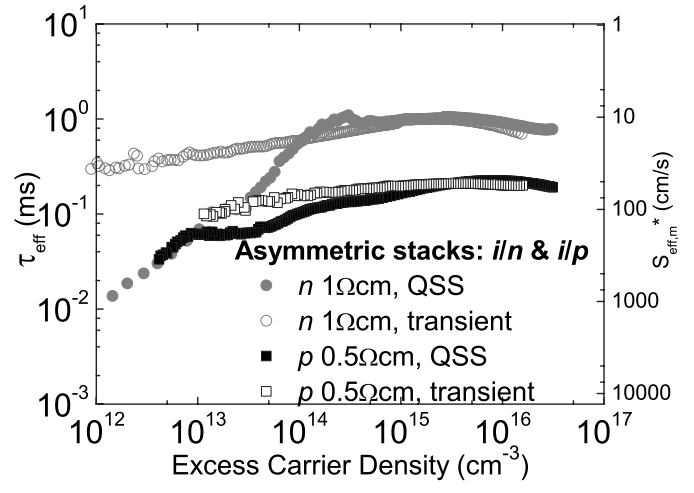

FIG. 15. Higher $n$ - and $p$-type doped c-Si surfaces passivated with asymmetric stacks of intrinsic plus doped a-Si:H layers. The QSS and transient measurements are distinguished by plain and empty symbols. These devices are the solar cell precursors whose cell performances after further contact formation are shown in Fig. 16. Note that $S_{\text {eff,m }}^{*}$ contains the information from the front and back sides.

well represented by our model, besides the low-level ECD discrepancies occurring between $S_{e f f, m}$ and $S_{e f f, c}$, which are due to our simple model's validity limits (see Sec. IV D 2).

\section{F. Heterojunction devices}

Finally, asymmetrically doped stacks of intrinsic plus doped layers are grown on both sides of higher doped $n$ - and $p$-type c-Si wafers. With a reduced $i$-layer thickness of $5 \mathrm{~nm}$, these structures result in heterojunction solar cells, simply by further transparent contact deposition. The injection level dependent interface passivation of the two cell precursors yielding the best cell performances is shown in Fig. 15. Measurements shown are made by illuminating the stack containing the layer of opposite doping type than the c-Si wafer, i.e., the front of the solar cell precursor. Transient measurements yield the same curves when illuminating from the other side (not shown in Fig. 15) proving the validity of the hypothesis of uniform carrier generation in the c-Si wafer. QSS and transient measured data are presented both as $\tau_{\text {eff }}(\mathrm{ECD})$ curves as originally measured by the Sinton lifetime tester, as for asymmetrical passivation, Eq. (8) is no longer valid. Nevertheless, on the right hand $y$ axis of Fig. 15, values for $S_{\text {eff }}$ as calculated from Eq. (8) are indicated to facilitate graphical comparison with the preceding figures. The so calculated $S_{e f f, m}^{*}$ corresponds roughly to the average of front and back passivations. The respective contribution of each of both asymmetrical surface passivation layers to $S_{e f f, m}^{*}$ could be evaluated by other lifetime measurement techniques such as the microwave-detected photoconductance decay. ${ }^{39}$ In contrast to microdoped passivation layers, the transient and not the QSS measurement mode yields lower $S_{e f f, m}^{*}$ values, i.e., better passivation quality. This observation can again be possibly related to the different a-Si:H/c-Si interface thicknesses analyzed by the transient and QSS measurement modes mentioned in Sec. IV D 1. The transient mode, analyzing the relaxed $5 \mathrm{~nm}$ thick $i$ a-Si:H layer, yields lower values for $S_{\text {eff }}$ than those obtained in the QSS mode, in which some of the 


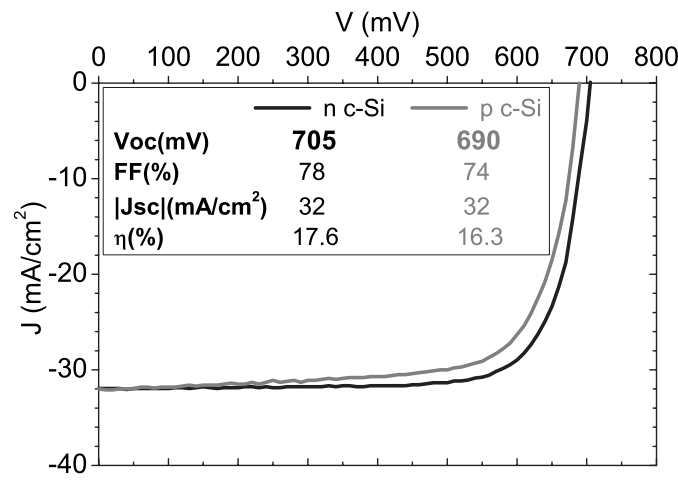

FIG. 16. Heterojunction solar cell characteristics on flat $n$-type and $p$-type doped c-Si. These are the devices whose precursor's passivation performances are shown in Fig. 15.

more defective doped layer is analyzed too. Together with the already observed poorer passivation performance of (even lightly) $p$-type doped a-Si:H layers, these observations could also explain the slight differences observed when comparing QSS data measured when illuminating the side with the stack including the $p$-type a-Si:H layer or when illuminating the other side including the $n$-type doped a-Si:H layer (not shown in Fig. 15).

As photoconductance in the stacked structure and device open-circuit voltage are both ultimately measurements of the same excess carrier density, a predicted open-circuit voltage $\left(V_{O C}\right)$ value can be calculated from the lifetime data. ${ }^{31}$ At 1 sun illumination, these are $V_{O C}=705 \mathrm{mV}$ on $n$-type c-Si and $V_{O C}=695 \mathrm{mV}$ on $p$-type c-Si for the solar cell stacked precursors shown in Fig. 15. Finally, in order to extract photocurrent out of these heterojunction structures, the stacked precursors are contacted by the deposition of indium tin oxide layers on both sides, followed by $\mathrm{Al}$ or $\mathrm{Ag}$ metallization on the back, and structured into about $0.2 \mathrm{~cm}^{2}$ area size solar cells. Final device performances are evaluated from currentvoltage $(I-V)$ curves under standard AM1.5 illumination and external quantum efficiency measurements. The resulting $I-V$ curves presented in Fig. 16 show that the $V_{O C}$ 's predicted by the passivation structure are reached. Thus, good solar cell electrical performances are achieved, even if a rather low current density is obtained due to the use of flat c-Si wafers (no light trapping).

\section{DISCUSSION}

We applied a simple dangling bond recombination model $^{11}$ to analyze a-Si:H/c-Si interface recombination. This approach is based on the fact that the silicon dangling bonds are the electronically active defects at the c-Si surface as well as in bulk a-Si:H. These defects posses an amphoteric nature; i.e., they can have three different states of charge. The resulting DB recombination rate differs considerably from the commonly used SRH recombination rate in undoped material and reduces to a SRH-like recombination rate in the case of doped layers. By introducing this recombination rate in the classical description of diffusion and recombination of photogenerated carriers in the interface layer, we are able to numerically reproduce the measured injection level dependence of the surface recombination velocity.

Discrepancies between model and measured data are identified as consequences of oversimplified assumptions made to keep our model as simple as possible. To model $S_{\text {eff, },}(\mathrm{ECD})$ on the intrinsic c-Si, thermal emission from recombination centers within the c-Si band gap into the a-Si:H has to be included to model the surface recombination increase at decreasing injection levels observed experimentally. One could, therefore, think that introducing this $\mathrm{c}-\mathrm{Si}$ thermal emission in our model is the reason why high values of $S_{\text {eff,c }}$ are predicted at low-level injection for c-Si/ microdoped and $\mathrm{c}-\mathrm{Si} / i /$ doped a-Si:H stacks. However, in fact, the higher the wafer doping, the smaller the surface potential and the more the effect on $S_{\text {eff.c }}$ of thermal emission from recombination centers within the c-Si band gap is negligible over the whole illumination level range. Thus, here, the introduction of c-Si thermal emission is not the reason for the strong increase of $S_{e f f, c}$ at low illumination levels. The observed flattening of $S_{e f f, m}$, indicative of a lower surface recombination than predicted by our model, can be explained by the fact that with decreasing illumination, fewer and fewer DB states act as recombination centers. This effect is related to the final (dark) position of the Fermi level within the density of states and the relative splitting of the quasiFermi-levels as function of time during the measurement. In $\mathrm{a}-\mathrm{Si} \mathrm{H}$, the more the Fermi level is distant from midgap, the greater the chance for one of the quasi-Fermi-levels of traps to lie still in the DB distribution at low-level illumination. Finally, the injection level dependent surface recombination velocity of the heterojunction device samples is not fitted because of their asymmetric passivation structure. However, the flattening of their $S_{e f f, m}^{*}(\mathrm{ECD})$ curves at low illumination can be identified to be the consequence of the same effects as described above. Note that in short-circuit conditions of a solar cell, $\Delta n$ is in the range of $10^{12}-10^{14}$ and, hence, the flattening is desirable. The sometimes observed bad overlap of different curve segments constituting the whole $S_{\text {eff, } m}(\mathrm{ECD})$ curve of one sample measured by photoconductance with two different measurement modes, made at different illumination levels and penetration depths, is explained by the different interface volumes analyzed in each mode.

By means of the quantification of the interface parameters $N_{S}$ and $Q_{S}$, the modeling allows us to conclude the following:

(1) $5 \mathrm{~nm}$ thick a-Si:H layers are not stable against ambient storage due to their outer surface potential modification.

(2) The density of the interface recombination centers $N_{S}$ decreases with the layer thickness due to the relaxation of the first grown monolayers to reach a minimal value for a-Si:H thickness of about $40 \mathrm{~nm}$. For thicker layers (up to $500 \mathrm{~nm}$ ), $N_{S}$ increases again due to the mechanical stress induced at the a-Si:H/c-Si interface.

(3) $n$-type microdoping of a-Si:H results, as expected, in a negative average dangling bond charge without noticeable variation of $N_{S}$. Surprisingly, $p$-type microdoping does not modify considerably the average positive DB charge state, already observed in $i$ a-Si:H passivating layers. It even lightly increases $N_{S}$, a well known detrimental effect of boron incorporation in the a-Si:H network. 
(4) Stacks of intrinsic plus doped a-Si:H layers show, as expected, the same effects as observed while varying the average DB charge state by microdoping.

The passivation performance of a-Si:H is validated by fabricating high- $V_{O C}$ heterojunction solar cells by means of asymmetric stacks grown on higher doped $n$-type as well as $p$-type c-Si. Intrinsic a-Si:H is therefore a high-performance passivation scheme for all kinds of c-Si materials due to (1) its low $N_{S}$ and (2) the almost symmetrical neutral capture cross sections for electrons and holes. Our interpretation of the lowest $S_{\text {eff, } m}$ obtained on $n$-type wafers passivated with a-Si:H layers is the following: as holes have a larger capture cross section than electrons $\left(\sigma_{p}^{0}>\sigma_{n}^{0}\right)$, the largest free-carrier lifetime for a given $N_{S}$ is to be expected when $n_{s}>p_{s}$ ( $n$-type wafer) and recombination occurs via neutral DBs.

If the effective surface recombination velocity $S_{\text {eff }}$ increases linearly with ECD, the surface passivation quality can be described by a single injection level independent parameter, the saturation current density $J_{0}$, that has to be minimized. If there is no linear increase in $S_{\text {eff }}$ with injection level, this is no longer possible. Until now, in such cases, no simple model had permitted to fit measured injection level dependence of $S_{\text {eff }}$ simultaneously on passivated intrinsic, $n$ and $p$-type doped c-Si wafers. Usually the extended SRH formalism is used to model the two standard c-Si surface passivation schemes $\mathrm{SiO}_{2}$ and $\mathrm{SiN}_{x}$. For this, it is necessary to determine the energy dependence of the capture cross sections $\sigma_{n, p}(E)$ and of the interface state density $D_{i t}(E)$ by deep level transient spectroscopy. ${ }^{40} \mathrm{Here}$, in our interface recombination model, in order to find the effective surface recombination velocity in Eqs. (4) and (6), we do not need to know the shape of the continuous distribution of DB states obtained resulting by other authors from the knowledge of the product of $\sigma_{n, p}(E) \times D_{i t}(E)$ over the whole energy range in the gap. ${ }^{8,40}$

The absolute lowest interface state density is obtained with thermal $\mathrm{SiO}_{2}$ passivation schemes. ${ }^{5}$ Due to its larger capture cross section for electrons than for holes, the passivation performance of $\mathrm{SiO}_{2}$ is superior on $n$-type c-Si. ${ }^{40}$ However, this passivation scheme suffers from long-term stability problems. Another widespread passivation scheme uses $\mathrm{SiN}_{x}$ passivation, which is dominated by field effect. Its large inherent positive charge leads to low minimal values of surface recombination velocity when passivating lightly doped c-Si wafers, but inferior device performance than obtained with $\mathrm{SiO}_{2} \cdot{ }^{41}$ Nevertheless, the injection level dependence of $S_{\text {eff }}$ at the $\mathrm{SiN}_{x} / \mathrm{c}-\mathrm{Si}$ interface is found to be astonishingly similar to the one at the $\mathrm{SiO}_{2} / \mathrm{c}-\mathrm{Si}$ interface. ${ }^{6}$ On one hand, Biegelsen et $\mathrm{al}^{3}$ already believed that there are common physical mechanisms underlying the characteristic attributes of the two systems a-Si:H and $\mathrm{SiO}_{2} / \mathrm{c}-\mathrm{Si}$ justified by their electron spin resonance (ESR) measurements. The amphoteric nature of bulk $\mathrm{SiN}_{x}$ was demonstrated somewhat later also by ESR measurements. ${ }^{4}$ On the other hand, the three different types of defects with broad Gaussian-like density distributions found at the $\mathrm{SiN}_{x} / \mathrm{c}-\mathrm{Si}$ interface ${ }^{6}$ can be made to coincide with our dangling bond interface recombination model by considering the appropriate set of recombination parameters for supplementary existing extrinsic DBs. Finally, from the similarities and various advantages and disadvan- tages of these different passivation schemes, it is becoming obvious that their use in combined stacks is a promising avenue for improved stable passivation schemes in the c-Si wafer industry. Recently, for example, a thin a-Si:H layer stack with a capping $\operatorname{SiN}_{x}$ layer has been shown to give excellent passivation of front diffused emitters, ${ }^{33}$ the $\mathrm{SiN}_{x}$ layer acting at the same time as an antireflection coating.

\section{CONCLUSION}

We have established a simple model for a-Si:H/c-Si interface recombination based on the amphoteric nature of silicon dangling bonds. Our model fits well to experimentally measured injection level dependent lifetimes on various combinations of intrinsic, microdoped, or internally polarized $i$ a-Si:H layers on a wide range of wafer doping $(p, n$, and intrinsic). It is a simple model that allows the fit of such a large set of combinations of wafers and passivation layers. The sturdiness of our model fundamentally relies on the unique properties of DBs to posses three states of charge, which was not taken into account within a simple model until now in this field. We are, thus, able to quantify the two parameters governing passivation, which are, on one hand, the density of interface recombination centers and, on the other hand, the passivation layer's average charge density, governing (by field effect) the depletion of one carrier type within the wafer. Therefore, we conclude that the growth of intrinsic a-Si:H on c-Si leads to a low interface recombination center density and that field-effect passivation can be tuned by varying the average state of charge of the interface's dangling bond recombination centers. a-Si:H passivation of c-Si is more symmetric, as far as field effect is concerned, than $\mathrm{SiO}_{2}$ and $\mathrm{SiN}_{x}$. According to our model, this is fundamentally related to the more symmetric microscopic parameters governing the recombination, which for a-Si:H are basically the neutral capture cross sections and the above mentioned tunability of field-effect passivation. Therefore, the passivation of $\mathrm{c}-\mathrm{Si}$ with a-Si:H has a broader range of applications. $\mathrm{SiO}_{2}$ is a better passivation layer on $n$-type c-Si compared to $p$-type c-Si, and this is due to markedly asymmetric capture cross sections for electrons and holes. In addition, $\mathrm{SiO}_{2}$ is not stable. $\mathrm{SiN}_{x}$ well passivates lightly doped c-Si wafers and is an excellent antireflection coating, but device performance of $\mathrm{SiN}_{x}$-passivated c-Si is limited by its high field-effect passivation. Considering all criteria such as thermal and long-term stabilities, optical performance, surface topography, and preconditioning, and understanding the underlying physics help to make the best choice of single or stacked passivation system for each application. ${ }^{42,43}$ The polyvalency of a-Si:H passivation is exploited here by fabricating high- $V_{O C}$ heterojunction solar cells on $n$-type as well as on $p$-type doped flat $\mathrm{c}$-Si.

\section{ACKNOWLEDGMENTS}

This work is supported by the Swiss National Foundation (FN-200021-107469). The authors thank H. Keppner for reading the manuscript and making helpful suggestions. 
*sara.olibet@unine.ch

${ }^{1}$ M. A. Green, F. D. King, and J. Shewchun, Solid-State Electron. 17, 551 (1974).

${ }^{2}$ R. Hezel and R. Schörner, J. Appl. Phys. 52, 3076 (1981).

${ }^{3}$ D. K. Biegelsen, N. M. Johnson, M. Stutzmann, E. H. Poindexter, and P. J. Caplan, Appl. Surf. Sci. 22/23, 879 (1985).

${ }^{4}$ D. T. Krick, P. M. Lenahan, and J. Kanicki, Phys. Rev. B 38, 8226 (1988).

${ }^{5}$ M. J. Kerr and A. Cuevas, Semicond. Sci. Technol. 17, 35 (2002).

${ }^{6}$ J. Schmidt and A. G. Aberle, J. Appl. Phys. 85, 3626 (1999).

${ }^{7}$ J. I. Pankove and M. L. Tarng, Appl. Phys. Lett. 34, 156 (1979).

${ }^{8}$ A. G. Aberle, Crystalline Silicon Solar Cells: Advanced Surface Passivation and Analysis (Centre for Photovoltaic Engineering, University of New South Wales, Sydney, 1999).

${ }^{9}$ M. Taguchi, H. Sakata, Y. Yoshimine, E. Maruyama, A. Terakawa, and M. Tanaka, Proceedings of the 31st IEEE Photovoltaic Specialists Conference, Florida, 2005, p. 866.

${ }^{10}$ F. Vaillant and D. Jousse, Phys. Rev. B 34, 4088 (1986).

${ }^{11}$ J. Hubin, A. V. Shah, and E. Sauvain, Philos. Mag. Lett. 66, 115 (1992).

${ }^{12}$ J. G. Simmons and G. W. Taylor, Phys. Rev. B 4, 502 (1971).

${ }^{13}$ R. S. Crandall, in Semiconductors and Semimetals (Academic, New York, 1984), Vol. 21B, p. 257.

${ }^{14}$ W. Shockley and W. T. Read, Phys. Rev. 87, 835 (1952).

${ }^{15}$ R. N. Hall, Phys. Rev. 87, 387 (1952).

${ }^{16}$ C. Longeaud, J. A. Schmidt, and R. R. Koropecki, Phys. Rev. B 73, 235317 (2006).

${ }^{17}$ A. Eray, G. Nobile, and F. Palma, Mater. Sci. Eng., B 102, 398 (2003).

${ }^{18}$ L. Korte, A. Laades, and M. Schmidt, J. Non-Cryst. Solids 352, 1217 (2006).

${ }^{19}$ M. Favre, H. Curtins, and A. V. Shah, J. Non-Cryst. Solids 97/98, 731 (1987)

${ }^{20}$ S. M. Sze, Semiconductor Devices: Physics and Technology, 2nd ed. (Wiley, New York, 2001).

${ }^{21}$ R. A. Street, Hydrogenated Amorphous Silicon (Cambridge University Press, Cambridge, 1991).

${ }^{22}$ M. Burgelman, P. Nollet, and S. Degrave, Thin Solid Films 361362, 527 (2000)
${ }^{23}$ M. Burgelman, J. Verschraegen, S. Degrave, and P. Nollet, Prog. Photovoltaics 12, 143 (2004).

${ }^{24}$ J. Hubin, A. V. Shah, E. Sauvain, and P. Pipoz, J. Appl. Phys. 78, 6050 (1995).

${ }^{25}$ R. Meaudre and M. Meaudre, Appl. Phys. Lett. 85, 245 (2004).

${ }^{26}$ N. Beck, N. Wyrsch, C. Hof, and A. Shah, J. Appl. Phys. 79, 9361 (1996).

${ }^{27}$ R. A. Street, J. Zesch, and M. J. Thompson, Appl. Phys. Lett. 43, 672 (1983).

${ }^{28}$ S. Tchakarov, U. Dutta, P. R. I. Cabarrocas, and P. Chatterjee, J. Non-Cryst. Solids 338-340, 766 (2004).

${ }^{29}$ R. A. Street, J. Non-Cryst. Solids 77/78, 1 (1985).

${ }^{30}$ Y. Yan, M. Page, T. H. Wang, M. M. Al-Jassim, H. M. Branz, and Q. Wang, Appl. Phys. Lett. 88, 121925 (2006).

${ }^{31}$ R. A. Sinton and A. Cuevas, Appl. Phys. Lett. 69, 2510 (1996).

${ }^{32}$ H. Fujiwara and M. Kondo, Appl. Phys. Lett. 86, 032112 (2005).

${ }^{33}$ H. Plagwitz, Y. Takahashi, B. Terheiden, and R. Brendel, Proceedings of the 21st European Photovoltaic Solar Energy Conference, Dresden, Germany, 2006, p. 688.

${ }^{34}$ M. Sebastiani, L. DiGaspare, G. Capellini, C. Bittencourt, and F. Evangelisti, Phys. Rev. Lett. 75, 3352 (1995).

${ }^{35}$ H. C. Neitzert, W. Hirsch, and M. Kunst, Phys. Rev. B 47, 4080 (1993).

${ }^{36}$ W. Wanlu and L. Kejun, Thin Solid Films 165, 173 (1988).

${ }^{37}$ S. DeWolf and G. Beaucarne, Appl. Phys. Lett. 88, 022104 (2006).

${ }^{38}$ M. Garín, U. Rau, W. Brendle, I. Martín, and R. Alcubilla, J. Appl. Phys. 98, 093711 (2005).

${ }^{39}$ K. L. Luke and L. J. Cheng, J. Appl. Phys. 61, 2282 (1987).

${ }^{40}$ A. G. Aberle, S. Glunz, and W. Warta, J. Appl. Phys. 71, 4422 (1992).

${ }^{41}$ J. Y. Lee and S. W. Glunz, Sol. Energy Mater. Sol. Cells 90, 82 (2006).

${ }^{42} \mathrm{~S}$. W. Glunz et al., Proceedings of the 20th European Photovoltaic Solar Energy Conference, Barcelona, Spain, 2005, p. 572.

${ }^{43}$ P. P. Altermatt, H. Plagwitz, R. Bock, J. Schmidt, R. Brendel, M. J. Kerr, and A. Cuevas, Proceedings of the 21st European Photovoltaic Solar Energy Conference, Dresden, Germany, 2006, p. 647. 